\title{
Remarkable regression of massive deep vein thrombosis in response to intensive oral rivaroxaban treatment
}

\author{
Norimichi Koitabashi ${ }^{*}$, Nogiku Niwamae ${ }^{2}$, Tetsuya Taguchi ${ }^{3}$, Yoshiaki Ohyama $^{1}$, Noriaki Takama ${ }^{1}$ \\ and Masahiko Kurabayashi ${ }^{1}$
}

\begin{abstract}
Deep vein thrombosis (DVT) is a common disease and is associated with pulmonary embolism (PE). Proximal iliofemoral DVT may lead to severe PE and chronic venous insufficiency. The standard therapy for DVT is anticoagulant therapy using heparin and a vitamin $\mathrm{K}$ antagonist, but a recent clinical study showed that rivaroxaban, an oral Xa inhibitor, was comparable to standard therapy and had less bleeding complications. Intensive high-dose anticoagulation is recommended during the initial 3 weeks of DVT treatment. The present report describes a case of a 77-year-old male showing a remarkable regression of DVT in response to rivaroxaban treatment within the initial 3 weeks of therapy and who did not experience any adverse events. His DVT was massive and was accompanied by proximal iliofemoral vein thrombus and iliac vein compression syndrome. Rivaroxaban, especially in intensive high-dose treatment, might be a safe and effective therapeutic choice for massive DVT.
\end{abstract}

Keywords: Rivaroxaban, Deep vein thrombosis, Anticoagulation, lliac vein compression, Thrombus regression

\section{Background}

Venous thromboembolism (VTE), including deep vein thrombosis (DVT) and pulmonary embolism (PE), is a common medical condition and is the third leading cause of cardiovascular death [1]. The clinical severity of DVT is associated with the burden and location of the venous thrombus. Patients with proximal iliofemoral DVT are most likely to have early and late morbidity [2]. Anticoagulant therapy is effective for the management of acute VTE and for the prevention of recurrent events and death associated with recurrent VTE $[1,3]$. For half a century, the standard therapy for patients with VTE has been the administration of heparin, overlapped and followed by a vitamin $\mathrm{K}$ antagonist (VKA) [1]. This regimen is effective but has several limitations, such as requirement for laboratory monitoring and dose adjustments, a narrow therapeutic window, and interaction with other drugs and foods [1,4]. Recently, new

\footnotetext{
* Correspondence: norikoitabashi@gmail.com

'Department of Medicine and Biological Sciences, Gunma University Graduate School of Medicine, 3-39-22, Showa-machi, Maebashi, Gunma 371-8511, Japan

Full list of author information is available at the end of the article
}

direct oral anticoagulants (DOACs), such as dabigatran, rivaroxaban, apixaban and edoxaban, which were developed for stroke prevention in the context of atrial fibrillation [5], have been shown to be effective for the treatment of VTE [6,7]. Rivaroxaban offers a simple, single-drug approach for initial intensive treatment, with high-dose rivaroxaban administered for 3 weeks followed by administration of standard dose rivaroxaban [8]. In the EINSTEIN-DVT [9] and EINSTEIN PE [4], this regimen showed no inferiority and no increase in adverse events when compared with standard therapy (low-molecular weight heparin [LMWH] with VKA).

Following these results, the J-EINSTEIN-DVT and -PE studies have been conducted in Japan [10]. These studies are open-label, randomized, multicenter trials that compared oral rivaroxaban alone with standard therapy in Japanese patients with DVT or PE. We participated in these studies and enrolled several patients. One patient with massive proximal DVT was assigned to rivaroxaban $15 \mathrm{mg}$ twice-daily and showed marked improvement during the first 3 weeks of therapy without any adverse effects including minor bleeding. 


\section{Case presentation}

A 77-year-old male was admitted to our hospital due to left lower limb swelling with heat sensation and redness. These symptoms had appeared 2 days prior to hospital admission. Five months before admission, he was diagnosed with polymyalgia rheumatica syndrome and started treatment with prednisolone $10 \mathrm{mg}$ per day. He had no previous history of thrombosis and no family history suggestive of inherited thrombophilia. His major risk for VTE was advanced age, but he did not have any other risks, such as obesity, cancer, surgery, immobilization or recent long travel. At the time of arrival to our Emergency Department, his entire left leg was swollen and showed pitting edema with mild pain. Blood pressure was $164 / 78 \mathrm{mmHg}$, and pulse of $102 \mathrm{bpm}$. Respiratory rate was $12 / \mathrm{min}$, and arterial oxyhemoglobin saturation was $96 \%$ at room air. Plasma D-dimer level was high $(45.0 \mu \mathrm{g} / \mathrm{mL})$ while serum protein level, renal function and liver function were normal. Multi-detector computed tomography (MDCT) showed massive deep vein thrombosis from the lower edge of inferior vena cava to the lower leg veins (Figure 1). This patient had a congenital anomaly of the iliac vein in which the left external iliac vein (EIV) and left internal iliac vein (IIV) separately branched off from the inferior vena cava without a left common iliac vein (Figure 1A). The left EIV was compressed by the abdominal aorta and the fifth lumbar vertebra (Figure 1A and B). The thrombosis extended from the calf veins to the compressed site, suggesting a variant of iliac vein compression syndrome (May-Thurner syndrome) [11,12]. The left IIV also had a large thrombus (Figure $1 \mathrm{C}$ and $\mathrm{D}$ ). The patient did not have any symptom related with $\mathrm{PE}$ and his lung MDCT did not show any embolism in the pulmonary arteries. Compression venous ultrasonography also showed massive DVT from the left iliofemoral level to the lower leg level, but slight venous flow was observed by color Doppler at the level of the left common femoral vein. A diagnosis of DVT was made, and the patient was immediately started on intravenous unfractionated heparin administration. The patient subsequently provided written informed consent to participate in the J-EINSTEIN study [10]. We screened thrombophilia including antiphospholipid syndrome, protein S/C deficiency, fibrinogen and antithrombin III abnormality, but could not find any cause of DVT except steroid administration. Then, prednisolone dose was reduced to $7.5 \mathrm{mg}$. Since the patient was assigned to begin rivaroxaban therapy on the second day of hospitalization, a 15-mg dose of rivaroxaban administration was given 4 hours after termination of heparin, according to the study protocol. His left leg swelling showed progressive reduction on a daily basis in response to rivaroxaban $15 \mathrm{mg}$ twice-daily. After 2 weeks of treatment, the patient was discharged from our hospital, as his symptoms and leg swelling had markedly improved. We did not use any compression therapy because the symptom improvement was observed from initial several days. The circumference of the left femoral region had decreased from $50.6 \mathrm{~cm}$ to $45.8 \mathrm{~cm}$ over the 2 weeks of initial treatment, and plasma D-dimer level decreased to $3.9 \mu \mathrm{g} / \mathrm{mL}$ by the time of hospital discharge. Surprisingly, the massive DVT in his left leg had almost completely disappeared according to MDCT performed on day 22 of the treatment (Figure 2). Compression venous ultrasonography at day 25 only showed a small thrombus at the left popliteal vein. As per protocol, we switched the dose of rivaroxaban to $15 \mathrm{mg}$ per day following 3 weeks of intensive therapy. We monitored his clinical symptom and physical examination every month and continued the study drug for 1 year. Neither bleeding nor recurrence of thrombosis occurred over the entire clinical course of the treatment.

\section{Discussion}

The present report described a case of a patient with DVT who showed marked improvement in response to oral rivaroxaban treatment during the initial intensive dose period. This patient had a massive thrombus extending from the left iliac vein to the lower leg veins with iliac vein compression syndrome. He had typical symptoms of DVT for 2 days but had no obvious risk factors for acute DVT except for steroid administration. $\mathrm{He}$ was enrolled in the J-EINSTEIN study and was assigned to receive rivaroxaban $15 \mathrm{mg}$ twice daily. After starting treatment, his leg edema progressive decreased on a daily basis. CT scan on day 22 revealed that the iliac vein thrombosis had almost completely disappeared. No bleeding event occurred during the course of treatment.

Iliac vein compression syndrome (May-Thurner syndrome) is most commonly seen in women at 30 to 50 years of age [12]. The right common iliac artery crosses over the left common iliac vein and then runs adjacent to the right iliac vein. Typically, the left iliac vein is compressed between the right common iliac artery anteriorly and the sacral promontory or the fifth lumbar vertebra posteriorly just before the iliocaval junction [12]. This case did not have a left common iliac vein and had venous return directly from the left external iliac vein to the vena cava at an unusually proximal position. We assume that this anomaly contributed to the development of iliac compression by the abdominal aorta. Aging-related atherosclerosis and vertebra deformity might anatomically augment the vein compression in this anomaly.

Proximal iliofemoral DVT causes PE in about half of cases. This condition can also lead to chronic venous insufficiency and recurrent thrombosis [2]. Compression of the iliac vein has been documented in about $50 \%$ of patients with left iliac DVT [12] and may require treatment via 

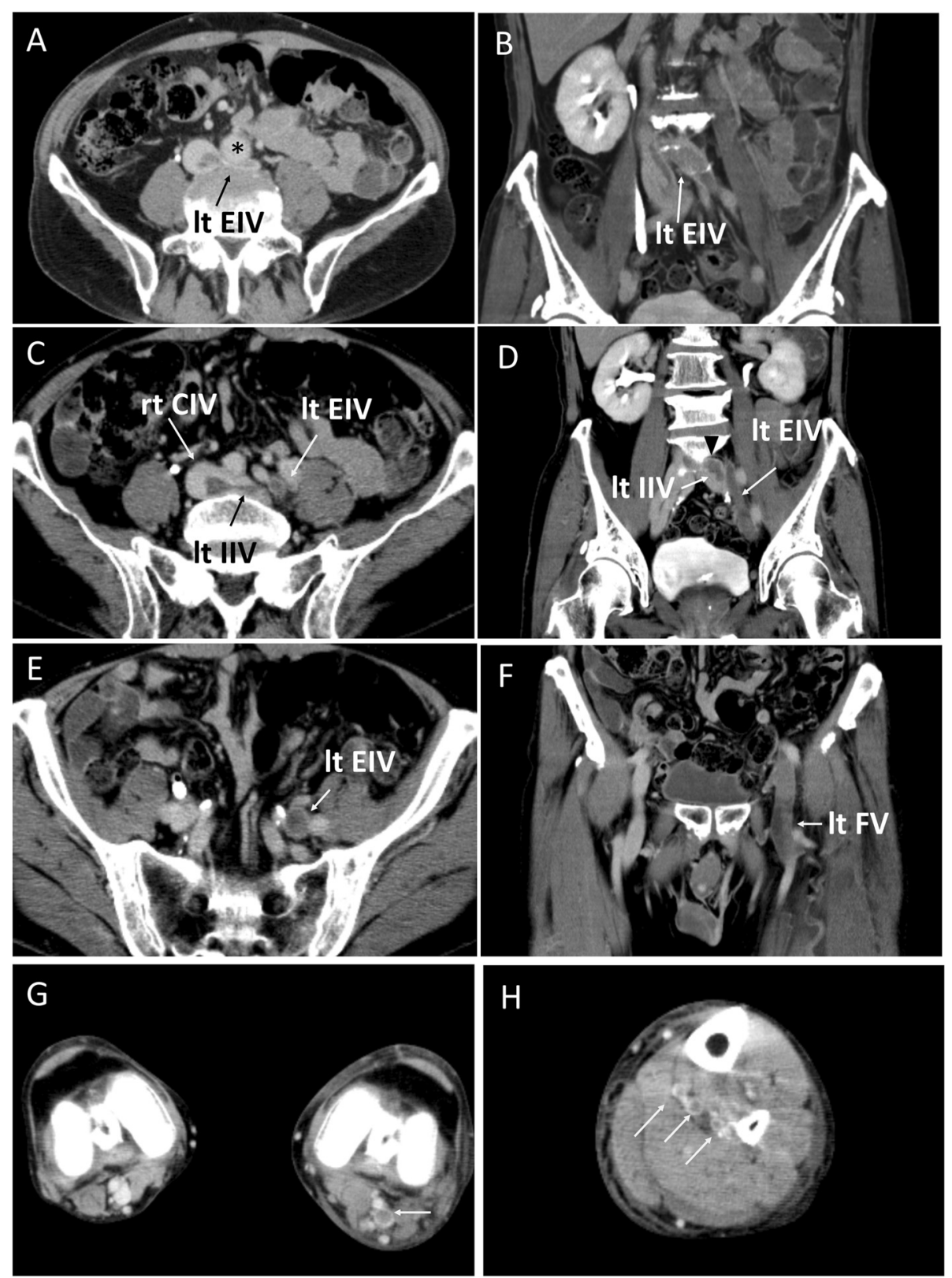

Figure 1 Multi-detector computed tomography (MDCT) on admission. A and $\mathbf{B}$. Transverse plane (A) and coronal plane (B) at the ilio-cava junction level. Left external iliac vein (It EIV) branches off directly from the inferior vena cava. The left EIA is compressed by the abdominal aorta $\left(^{*}\right)$ with thrombus; $\mathbf{C}$ to $\mathbf{E}$. Transverse plane ( $\mathbf{C}$ and $\mathbf{E}$ ) and coronal plane (D and $\mathbf{F}$ ) at the iliofemoral vein level. Lt EIV, right common iliac vein (rt CIV), left internal iliac vein (It IIV) and left femoral vein (let FV) have thrombi; G. left popliteal vein thrombus (white arrow); H. thrombi of left lower leg veins/soleal veins (white arrows).

invasive endovascular therapy, such as percutaneous balloon venoplasty and stent implantation [12,13]. However, some degree of compression of the left common iliac vein may also be an incidental finding [14]. Although our case was affected by the iliac vein compression, the decrease in thrombus size in response to medical therapy without the need for invasive approaches suggests that iliac vein compression did not have a significant effect on the response to medical therapy. After 3 weeks of high-dose treatment, the rivaroxaban dose was decreased to complete a 1-year course. The patient did not experience DVT recurrence despite persistent iliac compression.

Rivaroxaban is an oral Xa inhibitor that is as effective as standard anticoagulant therapy (i.e., heparin and warfarin) for VTE treatment $[4,9]$. Earlier studies showed that an intensive regimen ( $15 \mathrm{mg}$ twice-daily) during the first 3 weeks is necessary to achieve an effective higher trough levels and earlier steady state and thereby contribute to more effective 

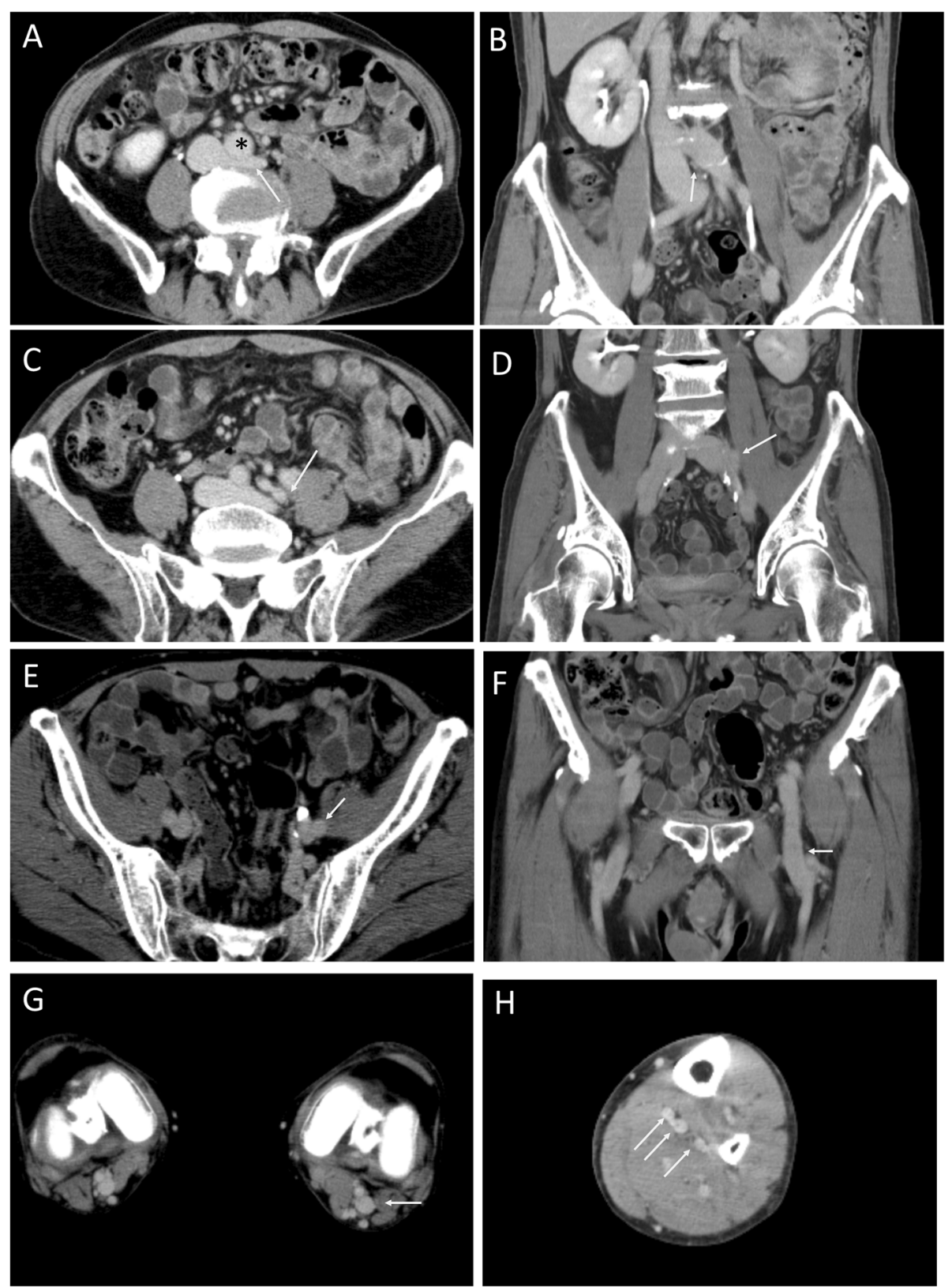

Figure 2 MDCT at day 22. A and B. Transverse plane (A) and coronal plane (B) at the ilio-cava junction level. * Abdominal aorta. Iliac vein compression persists, but the contrast defect has disappeared (white arrow); $\mathbf{C}$ to $\mathbf{E}$. Transverse plane (C and E) and coronal plane (D and F) at the iliofemoral vein level. Thrombi shown in Figure 1 have disappeared (white arrow); G. Absence of the left popliteal vein thrombus (white arrow); H. Absence of the left lower leg vein thrombus (white arrows).

regression of thrombosis [8]. The J-EINSTEIN study was designed to examine the effectiveness of rivaroxaban for Japanese patients with VTE [10]. A 30-mg dose per day is two times higher than the dose of standard anticoagulant therapy (15 mg per day) for atrial fibrillation in Japan. This high-dose intensive regimen, however, did not cause any significant adverse bleeding events, even in Japanese patients [10].
An important point in VTE treatment is to consider the risk and benefit of anticoagulant therapy, but the effectiveness of the selected therapy for thrombus regression should be considered as well $[2,3,15]$. Long-standing DVT related to insufficient anticoagulation might increase the risk of postthrombosis syndrome and organization of the thrombus $[2,15]$. Thrombolysis therapy is associated with a substantial effect on thrombus regression in massive 
DVT $[2,15]$, while bleeding risk is definitely increased in general $[16,17]$. Since the imaging technique for quantifying thrombus is limited, there was no comparable data for anticoagulant therapy in terms of ability for thrombus regression. Generally, standard anticoagulant therapy is not quite effective for thrombus regression when compared with thrombolysis therapy $[18,19]$. Although distal DVT, including those at the femoral level, may spontaneously regress with only anticoagulant therapy [19], the actual effect of the anticoagulant on DVT regression at the level of the iliac vein remains unclear. Surprisingly, the patient in the present case experienced nearly $100 \%$ thrombus regression over the first 3 weeks of therapy and did not experience recurrence, implying that the high-dose regimen might be better treatment than standard therapy.

In J-EINSTEIN study, regression of thrombus was more frequently observed in rivaroxaban-treated group compared with heparin-warfarin-treated group [10]. Khalafallah et al. showed a Caucasian case with a remarkable regression of bilateral extensive DVT by rivaroxaban suggesting that the efficacy would not be unique for Japanese patients [20]. However, since follow-up CT scan within a month from initial DVT treatment is generally uncommon in Western countries, there were few reports to show the early phase DVT regression induced by anticoagulant without thrombolysis. It would be worth to compare rivaroxaban and standard anticoagulants in terms of the thrombus-regressive effect in large international cohort in future.

There are several possible reasons for the marked effect of rivaroxaban in the present case. One reason is that the treatment was started during the early phase of DVT [3]. Another reason might be spontaneous recanalization [19], as compression venous ultrasonography showed slight venous flow even before treatment. Thrombus regression in response to anticoagulant therapy is more difficult to achieve in the context of an occluding thrombus. Recent several reports in left atrium or portal vein thrombosis indicate that rivaroxaban might be able to resolve clots rapidly [21-23]. A basic study showed that rivaroxaban-mediated suppression for thrombin generation leads a looser clot which is more degradable by fibrinolytic enzymes [24]. This mechanism may explain its efficacy in promoting the dissolution of the thrombus.

At present, the results of the trials using DOACs in the treatment of VTE showed that these agents are non-inferior and possibly safer than the standard heparinwarfarin regimen [3]. However, in several specific conditions such as cancer, pregnancy and renal insufficiency, the DOAC-use for VTE patents is limited [3]. Experience with DOACs in large patient populations is lacking so far and real-world patient outcome will need to be carefully monitored.

\section{Conclusions}

In summary, this report showed remarkable efficacy and safety of initial intensive treatment with rivaroxaban in a patient with massive thrombus. Further, no bleeding side effects were noted. While large-scale and precise investigation comparing standard treatment and new oral anticoagulants are required to validate these observations, this case suggests that rivaroxaban has the potential to replace conventional therapy with heparin and oral VKA for patients with massive DVT.

\section{Consent}

Written informed consent was obtained from the patient for publication of this Case report and any accompanying images.

\section{Abbreviations}

DOACs: Direct oral anticoagulants; DVT: Deep vein thrombosis; PE: Pulmonary embolism; VTE: Venous thromboembolism; MDCT: Multi-detector computed tomography; EIV: External iliac vein; IIV: Internal iliac vein; CIV: Common iliac vein.

\section{Competing interests}

This case was enrolled in the J-EINSTEIN trial. Publication of this case was approved by the J-EINSTEIN study group. This report was reviewed by Bayer HealthCare.

\section{Authors' contributions}

All authors were involved in the management of the patient. All authors contributed to the draft and revision of the manuscript and approved the final manuscript.

\section{Author details}

1 Department of Medicine and Biological Sciences, Gunma University Graduate School of Medicine, 3-39-22, Showa-machi, Maebashi, Gunma 371-8511, Japan. ${ }^{2}$ Department of Cardiovascular Medicine, Maebashi Red Cross Hospital, Maebashi, Gunma, Japan. '3epartment of Internal Medicine, Fukaya Red Cross Hospital, Fukaya, Saitama, Japan.

Received: 8 January 2015 Accepted: 6 March 2015

Published online: 14 March 2015

\section{References}

1. Goldhaber SZ, Bounameaux H. Pulmonary embolism and deep vein thrombosis. Lancet. 2012;379:1835-46.

2. Augustinos P, Ouriel K. Invasive approaches to treatment of venous thromboembolism. Circulation. 2004;110:127-34.

3. Konstantinides SV, Torbicki A, Agnelli G, Danchin N, Fitzmaurice D, Galie N, et al. 2014 esc guidelines on the diagnosis and management of acute pulmonary embolism: The task force for the diagnosis and management of acute pulmonary embolism of the european society of cardiology (esc)endorsed by the european respiratory society (ers). Eur Heart J. 2014;35:3033-73.

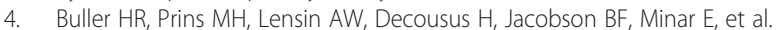
Oral rivaroxaban for the treatment of symptomatic pulmonary embolism. N Engl J Med. 2012;366:1287-97.

5. Schirmer SH, Baumhakel M, Neuberger HR, Hohnloser SH, van Gelder IC, Lip GY, et al. Novel anticoagulants for stroke prevention in atrial fibrillation: Current clinical evidence and future developments. J Am Coll Cardiol. 2010;56:2067-76.

6. Galanis T, Keiffer G, Merli G. The new oral anticoagulants for the treatment of venous thromboembolism: A new paradigm shift in antithrombotic therapy. Curr Ther Res Clin Exp. 2014;76:76-83.

7. Castellucci LA, Cameron C, Le Gal G, Rodger MA, Coyle D, Wells PS, et al. Clinical and safety outcomes associated with treatment of acute venous thromboembolism: A systematic review and meta-analysis. JAMA. 2014;312:1122-35.

8. Agnelli G, Gallus A, Goldhaber SZ, Haas S, Huisman MV, Hull RD, et al. Treatment of proximal deep-vein thrombosis with the oral direct factor xa 
inhibitor rivaroxaban (bay 59-7939): The odixa-dvt (oral direct factor xa inhibitor bay 59-7939 in patients with acute symptomatic deep-vein thrombosis) study. Circulation. 2007;116:180-7.

9. Bauersachs R, Berkowitz SD, Brenner B, Buller HR, Decousus H, Gallus AS, et al. Oral rivaroxaban for symptomatic venous thromboembolism. N Engl J Med. 2010;363:2499-510.

10. Yamada N, Hirayama A, Maeda H, Sakagami S, Shikata H, Prins MH, et al. Oral rivaroxaban for Japanese patients with symptomatic venous thromboembolism - the J-EINSTEIN DVT and PE program. Thromb J. 2015;13:2.

11. Brazeau NF, Harvey HB, Pinto EG, Deipolyi A, Hesketh RL, Oklu R. May-thurner syndrome: Diagnosis and management. Vasa. 2013;42:96-105.

12. Fazel R, Froehlich JB, Williams DM, Saint S, Nallamothu BK. Clinical problem-solving. A sinister development-a 35-year-old woman presented to the emergency department with a 2-day history of progressive swelling and pain in her left leg, without antecedent trauma. N Engl J Med. 2007;357:53-9.

13. Liu Z, Gao N, Shen L, Yang J, Zhu Y, Li Z, et al. Endovascular treatment for symptomatic iliac vein compression syndrome: A prospective consecutive series of 48 patients. Ann Vasc Surg. 2014;28:695-704.

14. Kibbe MR, Ujiki M, Goodwin AL, Eskandari M, Yao J, Matsumura J. lliac vein compression in an asymptomatic patient population. J Vasc Surg. 2004;39:937-43.

15. Goldhaber SZ, Meyerovitz MF, Green D, Vogelzang RL, Citrin P, Heit J, et al. Randomized controlled trial of tissue plasminogen activator in proximal deep venous thrombosis. Am J Med. 1990;88:235-40

16. Kanter DS, Mikkola KM, Patel SR, Parker JA, Goldhaber SZ. Thrombolytic therapy for pulmonary embolism. Frequency of intracranial hemorrhage and associated risk factors. Chest. 1997;111:1241-5.

17. Meyer G, Vicaut E, Danays T, Agnelli G, Becattini C, Beyer-Westendorf J, et al. Fibrinolysis for patients with intermediate-risk pulmonary embolism. N Engl J Med. 2014;370:1402-11.

18. Comerota AJ, Aldridge SC. Thrombolytic therapy for deep venous thrombosis: A clinical review. Can J Surg. 1993;36:359-64.

19. van Ramshorst B, van Bemmelen PS, Hoeneveld H, Faber JA, Eikelboom BC Thrombus regression in deep venous thrombosis. Quantification of spontaneous thrombolysis with duplex scanning. Circulation. 1992;86:414-9.

20. Khalafallah AA, Renu S, Sharp C, Hannan T. Extensive venous thrombosis in a healthy young man with a short inferior vena cava syndrome treated successfully with rivaroxaban. BMJ Case Rep. 2012; doi:10.1136/bcr-2012-007313.

21. Hammerstingl C, Potzsch B, Nickenig G. Resolution of giant left atrial appendage thrombus with rivaroxaban. Thromb Haemost. 2013;109:583-4.

22. Takasugi J, Yamagami H, Okata T, Toyoda K, Nagatsuka K. Dissolution of the left atrial appendage thrombus with rivaroxaban therapy. Cerebrovasc Dis. 2013;36:322-3.

23. Pannach S, Babatz J, Beyer-Westendorf J. Successful treatment of acute portal vein thrombosis with rivaroxaban. Thromb Haemost. 2013;1 10:626-7.

24. Varin R, Mirshahi S, Mirshahi P, Klein C, Jamshedov J, Chidiac J, et al. Whole blood clots are more resistant to lysis than plasma clots-greater efficacy of rivaroxaban. Thromb Res. 2013;131:e100-9.

\section{Submit your next manuscript to BioMed Central and take full advantage of:}

- Convenient online submission

- Thorough peer review

- No space constraints or color figure charges

- Immediate publication on acceptance

- Inclusion in PubMed, CAS, Scopus and Google Scholar

- Research which is freely available for redistribution 